\title{
SOBRE LA IDENTIDAD DE STAUROCLADIA CARPETANA Y MARSSONIELLA CARPETANA (CYANOPHYCEAE).
}

Palabras clave: Staurocladia, Marsoniella, Cyanophyceae.

\section{Antonio ROLDÁN y Marina ABOAL}

González Guerrero (1965) incluye en su trabajo «Las algas de la sílice» la descripción de un nuevo género y especie de cianofícea: Staurocladia carpetana, presente en muestras recolectadas en agosto de 1925 en la Laguna de Peñalara (Madrid). En este mismo trabajo hace referencia a Marssoniella carpetana descrita con anterioridad (González Guerrero, 1929).

Las características morfológicas de $S$. carpetana y $M$. carpetana se asemejan notoriamente a las de diversos conidios de hifomicetos acuáticos (Fungi Imperfecti, en particular a conidios del género Lemonniera De Wild.), hecho éste que ya apuntaba Bourrelly (1970) cuando sugiere la posible naturaleza fúngica de S. carpetana. Hay varios precedentes de confusión de conidios de hifomicetos acuáticos con algas en la literatura mundial. Así, conidios de Tetracladium marchalianum De Wildeman (1894) fueron descritos como una variedad del alga Cerasterias raphidioides Reinsch (Reinsch, 1888) y también fueron referidos por Printz (1914) al género Asterothrix. Otro ejemplo es Gyoerffyella rotula (Hoehnel) Marvanová (Marvanová Descals 1967), cuyos conidios fueron descritos por Kol (1928) como la Clorofícea Gyoerffyella tatrica.

Los conidios de hifomicetos acuáticos, debido a sus peculiares diseños adaptados al anclaje, pueden concentrarse en grandes cantidades entremezclados con briófitos y algas filamentosas, pero también aparecen en suspensión. De este modo, es posible su recolección involuntaria cuando se toman muestras de estas criptógamas.

Con estos antecedentes, se estimó conveniente una revisión del material original a partir del cual fueron descritas $S$. carpetana y M. carpetana (González Guerrero no estableció tipo para estas especies). Las muestras fijadas con formaldehido se conservan en tubos de ensayo, depositadas por el propio autor en el Herbario MA, aunque sin número de registro. Se realizaron varias preparaciones con el perifiton en suspensión y con conidios aislados con pipetas capilares. Posteriormente se tiñeron con lactofucsina. Estas preparaciones fijas se depositaron en el herbario MUB ( ${ }^{\circ}$ 999).

Un listado completo de las algas presentes en estas muestras se puede encontrar en González Guerrero (1927, 1929, 1965). Con respecto al grupo de las Cianofíceas solamente se ha detectado alguna filamentosa como Tolypothrix distorta (Fl.Dm.) Kütz. epífita sobre los briófitos, pero en ningún caso cianofíceas planctónicas. Sin embargo, con relativa frecuencia se han identificado conidios de varios hifomicetos acuáticos: Articulospora tetracladia Ingold, Heliscus lugdunensis Sacc. \& Thérry, Lemonniera cornuta Ranzoni (Fig. 1-3), L. terrestris Tubaki (Fig. 4) y Tricladium splendens Ingold. Todas estas especies pueden ser frecuentes en aguas ácidas y han sido citadas 
repetidas veces en España, incluida la Sierra de Guadarrama en Madrid (Descals y Manjón, 1989).

Especial atención merecen los conidios de L. cornuta detectados. Como se aprecia en las figuras (2-3), son cruciformes, constituidos por cuatro brazos curvados en distintos planos, opuestos por pares, con dimensiones de 30-45 x 3.5-4.5 $\mu \mathrm{m}$. El número de septos es variable y aumenta con la edad del conidio, hasta un máximo de 4(5) por brazo. La cicatriz de secesión se encuentra en la intersección de los brazos y no suele ser visible. En preparaciones sobre portaobjetos la curvatura tan característica de los brazos puede desaparecer (o quedar muy disimulada) por aplastamiento bajo el cubreobjetos (Figs. 1-3), quedando todo el conidio en un solo plano.

González Guerrero (1965, lámina IB) ilustra varios ejemplares de Staurocladia carpetana que se diferencian poco de los conidios de Lemonniera cornuta, identificados ahora en sus muestras. El protólogo establecido para esta especie es el siguiente:

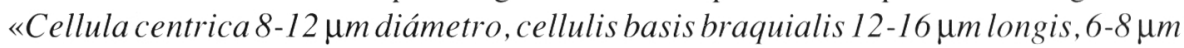
latis, vacuolatis caeruleo, tenui, sine granulis refringentibus in illis ad genicula non constrictis».

En el texto, González Guerrero señala que los brazos están dispuestos en un solo plano, posiblemente porque la observación microscópica en preparaciones sobre portaobjetos no permite apreciar su verdadera estructura tridimensional. Las dimensiones de las células basales dadas para $S$. carpetana son de 12-16 x 6-8 $\mu \mathrm{m}$. Con esta referencia se calcularon las dimensiones de los brazos del único ejemplar completo ilustrado por González Guerrero (Lam. IB, sin escala en el original), que son de 40-51 x 6-8 $\mu \mathrm{m}$. Estas dimensiones se asemejan a las de los conidios maduros de L. cornuta, salvo en la anchura de los brazos, que es algo mayor en la especie de González Guerrero. Desafortunadamente, es imposible la obtención de material autentificado de $S$. carpetana, pero teniendo en cuenta el artículo número 9.4 del Código Internacional de Nomenclatura Botánica (Greuter, 1988), se puede considerar como tipo el icón presentado por González Guerrero. Se considera, por tanto, lícito proponer Staurocladia carpetana González Guerrero, 1965 como species dubia.

Por otra parte, Komarek \& Vavra (1968) comprobaron que Marssoniella elegans no pertenecía al grupo de las cianofíceas, sino que se trataba de una agrupación de esporas de Gurleya marssoniella Vavra (Protozoa) y propusieron la desaparición del género Marssoniella.

Marssoniella carpetana fue descrita por González Guerrero (1929) con el siguiente protólogo: «Colonis radiaris-caespitosis caeruleo-viridibus, 4 cellularibum, cellulis periphericis piriformibus, 11.60-14.50 ×6.12-5.80 $\mu \mathrm{m}$, central unica quadrata 3.62-4.35 $\mu$ m diametro». En la muestra original no se ha observado ninguna cianofícea con estas características, aunque sí algunos conidios de Lemonniera terrestris Tubaki (1958) (Fig. 4). Esta especie se diferencia de L. cornuta en la disposición espacial de los brazos (difícilmente observable en preparaciones sobre portaobjetos) y en que los brazos del conidio son acuminados desde la base. Las dimensiones de los conidios de L. terrestris son muy variables, y en su rango inferior, pueden coincidir con las dadas 
por González Guerrero para M. carpetana. Los brazos del conidio de L. terrestris ilustrado en la figura 4 tiene 13-15 x 5.5-6.5 $\mu \mathrm{m}$. La morfología de $M$. carpetana en los dibujos que acompañan al protólogo es ligeramente diferente, aunque en González Guerrero (1965, Lam. IA) se ilustra un ejemplar de M. carpetana con morfología idéntica al conidio de L. terrestris de nuestra figura 4.

En consecuencia, se propone Marssoniella carpetana González Guerrero, 1929 como species dubia.

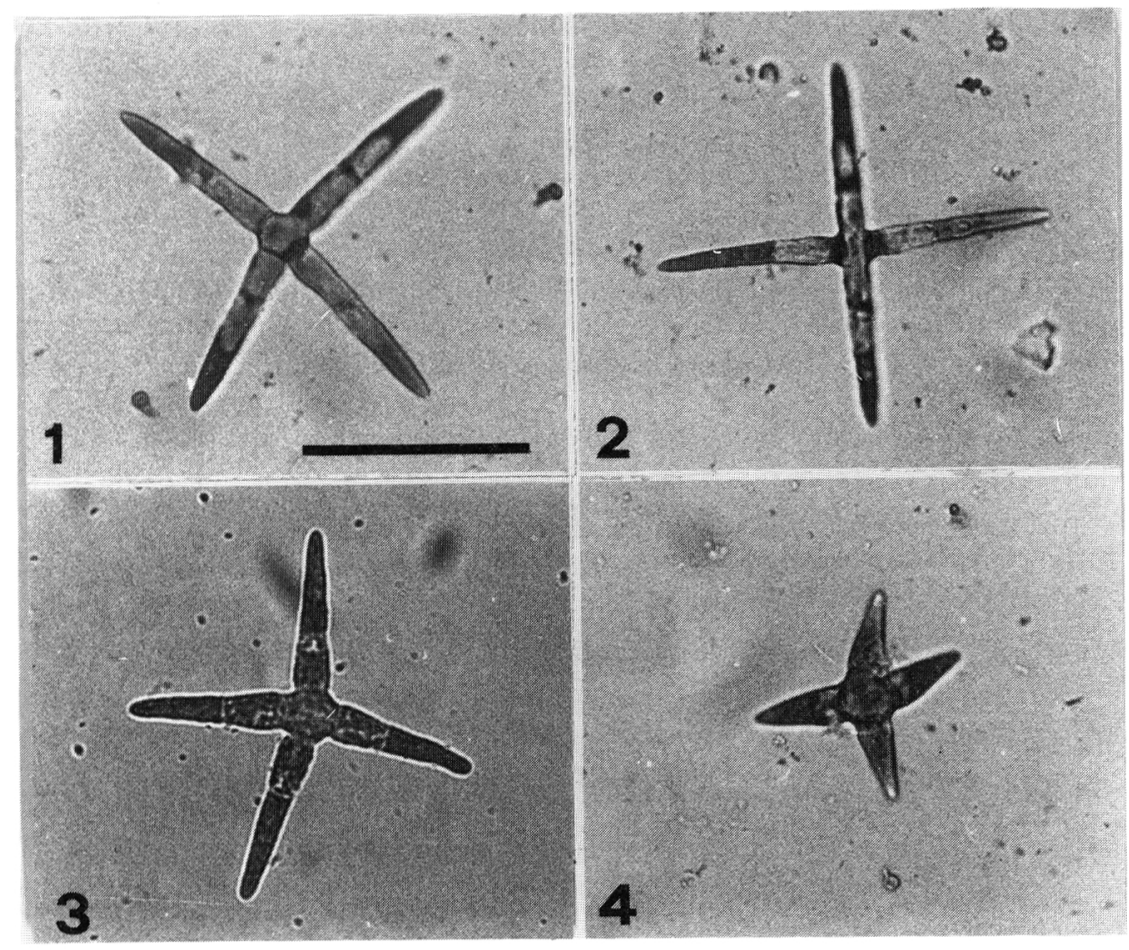

FIGURAS 1-4: Algunos conidios encontrados en las muestras de González Guerrero. 1-2.Lemonniera cornuta. 3.- Forma dudosa con características de L. cornuta y L. terrestris. 4.Lemonniera terrestris. Tinción con lactofucsina. La escala representa $40 \mu \mathrm{m}$.

\section{BIBLIOGRAFÍA}

BOURRELLY, P. 1-970- Les algues d'eau douce. Algues bleues et rouges. Boubée. París. DE WILDEMAN, E. - 1894- Notes mycologiques. Annales de la Societé belge de Microscopie, 18:135-161.

DESCALS, E. y J.L. MANJÓN -1989- Hongos acuáticos ingoldianos en la Sierra de

Guadarrama. Bol. Soc. Micol. Madrid, 13:11-26. 
GONZÁLEZ GUERRERO, P. -1927- Contribución al estudio de las algas y Esquizófitas de España. Trabajos del Museo Nacional de Ciencias Naturales. Serie Botánica, 22:1-52. GONZÁlEZ GUERRERO, P. -1929- Nuevos datos de plancton hispano-marroquí (agua dulce). Bol. R. Soc. Esp. Hist. Nat., 29:251-254.

GONZÁLEZ GUERRERO, P. -1965- Las algas de la sílice. Anals. Inst. Bot. A.J. Cavanilles, XXIII: $107-143$

GREUTER, W. (ed.). -1988-International Code of Botanical Nomenclature. XIV International Botanical Congress. Berlín 1987. Cramer.

KOL, E. -1928- Ueber die Kryovegetation der Hohen-Tátra. I. Folia Cryptog., 1:614-622.

VAVRA, J. \& J. VAVRA -1968- In memoriam Marssoniella Lemm. 1900. Arch. Protistenk., 3:12-17.

MARVANOVA, L., P. MARVAN, \& J. RUZICKA -1967- Gyoerffyella Kol. 1928, a genus of the hyphomycetes. Persoonia, 5(1):29-44.

PRINTZ, H. -1914- Kristianatraktens Protococcoideer. Skrifter udgat Videnskabsselskabet $i$ Christiania Mathematik-Natur. KL, 6:1-123.

RANZONI, F.V. 1-953- The aquatic hyphomycetes of California. Farlowia, 4:353-398.

PRINTZ, P.F. -1888- Familiae Polyedriarum Monographia. Notarisia, 3:493-516.

TUBAKI, K. -1958- Studies on Japanese hyphomycetes V. Leaf and stem group with a discussion of the classification of hyphomycetes and their perfect stages. J. Hatt. Bot. Lab., 20:142-244.

(Aceptado para su publicación en junio de 1991)

Dirección de los autores: A. Roldán: Unidad de recursos naturales. CEBAS-CSIC. Apdo. 4195. E-30080 Murcia. M. Aboal: Departamento de Biología Vegetal (Botánica). Facultad de Biología. Universidad de Murcia. E-30100. Murcia.

\section{CONTRIBUCIÓN A LA COROLOGÍA DE LOS BRIÓFITOS DE GALICIA}

Palabras clave. Briófitos, Galicia.

\section{Juan REINOSO FRANCO y $\mathbf{M}^{\mathrm{a}}$ del Carmen VIERA BENÍTEZ}

Continuando con nuestros estudios acerca de la flora briológica gallega, reseñamos en esta nota la presencia en la región de 6 nuevas especies y se confirman otras 8 citadas anteriormente en la bibliografía que constituyen novedad para la provincia de Lugo. Las nuevas citas para Galicia se señalan con un asterisco $(*)$.

Brachythecium velutinum (Hedw.) B.S.G.

Orbazay (Lugo) 29TPH1563. Sobre roca granítica. (SANT-bryo. 613). En Galicia sólo se conocía la referencia de Reinoso (1984) para la provincia de La Coruña, constituye, por lo tanto, primera cita para la provincia de Lugo. 